\title{
General Eulerian Numbers and Eulerian Polynomials
}

\author{
Tingyao Xiong, ${ }^{1}$ Hung-Ping Tsao, ${ }^{2}$ and Jonathan I. Hall ${ }^{3}$ \\ ${ }^{1}$ Department of Mathematics and Statistics, Radford University, Radford, VA 24141, USA \\ ${ }^{2}$ Department of Decision Sciences, San Francisco State University, CA 94132, USA \\ ${ }^{3}$ Department of Mathematics, Michigan State University, East Lansing, MI 48824, USA \\ Correspondence should be addressed to Tingyao Xiong; txiong@radford.edu
}

Received 26 October 2012; Accepted 3 January 2013

Academic Editor: Mike Tsionas

Copyright (C) 2013 Tingyao Xiong et al. This is an open access article distributed under the Creative Commons Attribution License, which permits unrestricted use, distribution, and reproduction in any medium, provided the original work is properly cited.

We will generalize the definitions of Eulerian numbers and Eulerian polynomials to general arithmetic progressions. Under the new definitions, we have been successful in extending several well-known properties of traditional Eulerian numbers and polynomials to the general Eulerian polynomials and numbers.

\section{Introduction}

Bernoulli [1, pages 95-97] had introduced his famous Bernoulli numbers, denoted by $B_{2 r}\left(B_{2 r+1}=0\right.$ for $\left.r \geq 1\right)$ to evaluate the sum of the $n$th power of the first $m$ integers. He then proved the following summation formula:

$$
\begin{aligned}
\sum_{i=1}^{m} i^{n}= & \frac{m^{n+1}}{n+1}+\frac{m^{n}}{2} \\
& +\frac{1}{n+1} \sum_{r=1}^{\lfloor r / 2\rfloor}\left(\begin{array}{c}
n+1 \\
2 r
\end{array}\right) m^{n-2 r+1}(-1)^{r+1} B_{2 r}
\end{aligned}
$$

when $n, m \geq 1$.

Two decades later, Euler [2] studied the alternating sum $\sum_{i=1}^{m}(-1)^{i} i^{n}$. He ended up with giving the following general result [3, (2.8), page 259]:

$$
\begin{aligned}
\sum_{i=1}^{m} i^{n} t^{i}= & \sum_{l=1}^{n}(-1)^{n+l}\left(\begin{array}{c}
n \\
l
\end{array}\right) \frac{t^{m+1} m^{l}}{(t-1)^{n-l+1}} A_{n-l}(t) \\
& +(-1)^{n} \frac{t\left(t^{m}-1\right)}{(t-1)^{n+1}} A_{n}(t) .
\end{aligned}
$$

Another simplified form of $\sum_{i=1}^{m} i^{n} t^{i}$ is the following [3, (3.3), page 263]:

$$
\begin{aligned}
\sum_{i=1}^{m} i^{n} t^{i}= & -t^{m+1} \sum_{k=0}^{n}\left(\begin{array}{l}
n \\
k
\end{array}\right) \frac{m^{n-k}}{(1-t)^{k+1}} A_{k}(t) \\
& +\frac{t}{(1-t)^{n+1}} A_{n}(t),
\end{aligned}
$$

where $A_{n}(t),(n=0,1,2, \ldots)$ are called Eulerian polynomials and are recursively defined by [3, (2.7), page 264]:

$$
A_{0}(t):=1,
$$

$$
A_{n}(t)=\sum_{k=0}^{n-1}\left(\begin{array}{l}
n \\
k
\end{array}\right) A_{k}(t)(t-1)^{n-1-k}
$$

Note that for $\sum_{i=1}^{m} i^{n} t^{i}$, if we put $t=-1$, then $\sum_{i=1}^{m} i^{n} t^{i}$ becomes the alternating sum of the $n$th power of the first $m$ positive integers. Furthermore, as $m \rightarrow \infty$, we have [3, (3.2), page 263]:

$$
\frac{A_{n}(t)}{(1-t)^{n+1}}=\sum_{j=0}^{\infty} t^{j}(j+1)^{n} \quad(n \geq 0) .
$$


Each Eulerian polynomial can be presented as a generating function of Eulerian numbers $A_{n, k}[4]$, also introduced by Euler, as

$$
A_{n}(t)=\sum_{k=0}^{n-1} A_{n, k} t^{k}
$$

Furthermore, the corresponding exponential generating function [3, (3.1), page 262] is

$$
A(t, u)=\sum_{n \geq 0} A_{n}(t) \frac{u^{n}}{n !}=\frac{t-1}{t-\exp (u(t-1))} .
$$

The following combinatorial definition of Eulerian numbers was discovered by Riordan in the 1950s.

Definition 1. A Eulerian number $A_{n, k}$ is the number of permutations $p_{1} p_{2} p_{3} \ldots p_{n}$ of the first $n$ numbers $\{1,2, \ldots, n\}$ that have $k$ ascents (or descents), that is, $k$ places where $p_{j}<$ $p_{j+1}\left(\right.$ or $\left.p_{j}>p_{j+1}\right), 1 \leq j \leq n-1$.

Example 2. When $n=3, k=1$, and $A_{3,1}=4$, because there are four permutations with only one ascent:

$$
132,213,231,312 \text {, }
$$

then $A_{n, k}$ satisfies the recurrence: $A_{n, 0}=1,(n \geq 0), A_{n, k}=0$ $(k \geq n)$ and

$$
\begin{aligned}
A_{n, k}= & (k+1) A_{n-1, k} \\
& +(n-k) A_{n-1, k-1} \quad(1 \leq k \leq n-1) .
\end{aligned}
$$

It is well known that Eulerian numbers have the following symmetric property:

Proposition 3. Given a positive integer $n$, and $0 \leq k \leq n-1$, $A_{n, k}=A_{n, n-1-k}$.

Proof. It is obvious because if a permutation has $k$ ascents then its reverse has $n-1-k$ ascents.

Furthermore, the values of $A_{n, k}$ can be expressed in a form of a triangular array as shown in Table 1.

Besides the recursive formula (9), $A_{n, k}$ can be calculated directly by the following analytic formula [3, (3.5), page 264]:

$$
\begin{array}{r}
A_{n, k}=\sum_{i=0}^{k}(-1)^{i}(k-i+1)^{n}\left(\begin{array}{c}
n+1 \\
i
\end{array}\right) \\
(0 \leq k \leq n-1) .
\end{array}
$$

Since the 1950s, Carlitz $([5,6])$ and his successors have generalized Euler's results to $q$-sequences $\left\{1, q, q^{2}, q^{3}, \ldots\right\}$. Under Carlitz's definition, the $q$-Eulerian numbers $A_{n, k}(q)$ are given by

$$
[x]^{n}=\sum_{k=0}^{n-1} A_{n, k}(q)\left[\begin{array}{c}
x+k-1 \\
n
\end{array}\right] \quad(n \geq 1),
$$

TABLE 1: First few values of Eulerian numbers $A_{n, k}$.

\begin{tabular}{rcccccr}
\hline & & \multicolumn{5}{c}{$k=$} \\
& 0 & 1 & 2 & 3 & 4 & 5 \\
\hline$n=1$ & 1 & & & & & \\
2 & 1 & 1 & & & & \\
3 & 1 & 4 & 1 & & & \\
4 & 1 & 11 & 11 & 1 & & \\
5 & 1 & 26 & 66 & 26 & 1 & \\
6 & 1 & 57 & 302 & 302 & 57 & 1 \\
\hline
\end{tabular}

where

$$
\begin{gathered}
{[x]=\frac{1-q^{x}}{1-q}} \\
{\left[\begin{array}{l}
x \\
n
\end{array}\right]=\frac{\left(1-q^{x}\right)\left(1-q^{x-1}\right) \cdots\left(1-q^{x-n+1}\right)}{(1-q)\left(1-q^{2}\right) \cdots\left(1-q^{n}\right)} .}
\end{gathered}
$$

Then the $q$-Eulerian polynomials $A_{n}(t, q)$ are defined as

$$
A_{n}(t, q)=\sum_{k=0}^{n-1} A_{n, k} t^{k} \quad(n \geq 1) .
$$

Like the traditional Eulerian numbers, the $q$-Eulerian numbers $A_{n, k}(q)$ have the following recursive formula:

$$
\begin{aligned}
& A_{1,0}(q)=1, \quad A_{n, k}(q)=0 \quad \text { if }(k \leq 0 \text { or } k \geq n), \\
& A_{n, k}(q)= q^{n-1-k}[k+1] A_{n-1, k}(q) \\
&+[n-k] A_{n-1, k-1}(q) \quad(n \geq 1) .
\end{aligned}
$$

Similarly to the traditional Eulerian numbers, we can also construct a triangular array for $q$-Eulerian numbers as in Table 2.

Definition 4. Given a permutation $\pi=p_{1} p_{2} p_{3} \ldots p_{n}$ of the first $n$ numbers $\{1,2,3, \ldots, n\}$, define functions

$$
\operatorname{maj} \pi=\sum_{p_{j}>p_{j+1}} j
$$

$a(n, k, i)=\#\{\pi \mid$ maj $\pi=i$ and $\pi$ has $k$ ascents $\}$.

In 1974, Carlitz [6] completed his study of his $q$-Eulerian numbers by giving a combinatorial meaning to his $q$-Eulerian numbers:

$$
A_{n, k}(q)=q^{((m-k+1)(m-k)) / 2} \sum_{i=0}^{k(n-k-1)} a(n, n-k, i) q^{i}
$$

where functions $a(n, k, i)$ are as defined in Definition 4 . Interested readers can find more details about the history of Eulerian numbers, Eulerian polynomials, and the corresponding concepts in q-environment from [3].

In this paper, instead of studying $q$-sequences, we will generalize Euler's work on Eulerian numbers and Eulerian polynomials to any general arithmetic progression:

$$
\{a, a+d, a+2 d, a+3 d, \ldots\} .
$$


TABLE 2: First values of $q$ - Eulerian numbers $A_{n, k}(q)$.

\begin{tabular}{rcccr}
\hline \multicolumn{5}{c}{$k=$} \\
& 0 & 1 & 2 & 3 \\
\hline$n=1$ & 1 & 1 & & \\
2 & $q$ & $2 q^{2}+2 q$ & 1 & \\
3 & $q^{3}$ & $3 q^{5}+5 q^{4}+3 q^{3}$ & $3 q^{3}+5 q^{2}+3 q$ & 1 \\
4 & $q^{6}$ & & & \\
\hline
\end{tabular}

In Section 2, we will give a new definition of general Eulerian numbers based on a given arithmetic progression as defined in (17). Under the new definition, some well-known combinatorial properties of traditional Eulerian numbers become special cases of our more general results. In Section 3 , we will define general Eulerian polynomials. Then, (2), (3), (4), (5), (6), (7), (9), and (10) become special cases of our more general results.

\section{General Eulerian Numbers}

The traditional Eulerian numbers $A_{n, k}$, play an important role in the well-known Worpitzky's identity [7]:

$$
x^{n}=\sum_{k=0}^{n-1}\left(\begin{array}{c}
x+k \\
n
\end{array}\right) A_{n, k}, \quad n \geq 1 .
$$

Before we give a general definition of Eulerian numbers based on a given arithmetic progression (17), we shall mention a property associated with the traditional Eulerian numbers $A_{n, k}$.

Proposition 5. Let $A_{n, k}$ be the traditional Eulerian numbers as defined in Definition 1, then

$$
\sum_{i=1}^{m} i^{n}=\sum_{k=0}^{n-1} A_{n, k}\left(\begin{array}{c}
m+k+1 \\
n+1
\end{array}\right) .
$$

Proof. See [8, (4), page 348].

Given an arithmetic progression (17), we want to define general Eulerian numbers $A_{n, k}(a, d)$ so that the important properties of traditional Eulerian numbers such as the recursive formula (9), the triangular array (Table 1), Worpitzky's Identity (18), and Proposition 5, and so forth, become special cases of more general results under the new definition.

Definition 6. General Eulerian numbers $A_{n, k}(a, d)$ associated with an arithmetic progression as in (17) are defined as $A_{0,-1}=1, A_{n, k}=0(k \geq n$ or $k \leq-2)$ and

$$
\begin{aligned}
A_{n, k}(a, d)= & (-a+(k+2) d) A_{n-1, k}(a, d) \\
& +(a+(n-k-1) d) A_{n-1, k-1}(a, d) \\
& (0 \leq k \leq n-1) .
\end{aligned}
$$

Like the traditional Eulerian numbers and $q$-Eulerian numbers, the first general Eulerian numbers can be presented in the form of a triangular array as in Table 3.
We intentionally choose $k$ values to start with -1 , because by doing so Table 1 becomes the special case of Table 3 when $a=d=1$ in the arithmetic progression:

$$
\{a, a+d, a+2 d, a+3 d, \ldots\} .
$$

In other words, Table 1 corresponds to the sequence of natural numbers:

$$
\{1,2,3,4, \ldots\} \text {. }
$$

Therefore when $a=d=1$, the entries in the first column of Table 3 become zeroes except initially defined $A_{0,-1}(a, d)=1$.

With the new Definition 6 , we are able to prove the following two properties. Again note that if $a=d=1$, then the following identity is just the conventional Worpitzky's identity.

Lemma 7 (General Worpitzky's Identity). Given an arithmetic progression as in (17),

$$
(a+(i-1) d)^{n}=\sum_{j=-1}^{n-1} A_{n, j}(a, d)\left(\begin{array}{c}
i+j \\
n
\end{array}\right), \quad i \geq 1 .
$$

Proof. We will prove Lemma 7 by induction on $n$.

(i) When $n=1$, using the values in Table 3,

$$
\begin{aligned}
a+(i-1) d= & A_{1,-1}(a, d)\left(\begin{array}{c}
i-1 \\
1
\end{array}\right) \\
& +A_{1,0}(a, d)\left(\begin{array}{l}
i \\
1
\end{array}\right) .
\end{aligned}
$$

(ii) Now suppose

$$
(a+(i-1) d)^{n}=\sum_{j=-1}^{n-1} A_{n, j}(a, d)\left(\begin{array}{c}
i+j \\
n
\end{array}\right) \text { then, }
$$

$$
\begin{aligned}
(a+ & (i-1) d)^{n+1} \\
= & {\left[\sum_{j=-1}^{n-1} A_{n, j}(a, d)\left(\begin{array}{c}
i+j \\
n
\end{array}\right)\right](a+(i-1) d) } \\
= & \sum_{j=-1}^{n-1} A_{n, j}(a, d)\left[a\left(\begin{array}{c}
i+j \\
n
\end{array}\right)-(j+2) d\left(\begin{array}{c}
i+j \\
n
\end{array}\right)\right. \\
& \left.+(i+j+1) d\left(\begin{array}{c}
i+j \\
n
\end{array}\right)\right] \\
= & \sum_{j=-1}^{n-1} A_{n, j}(a, d)\left[a\left(\begin{array}{c}
i+j+1 \\
n+1
\end{array}\right)-a\left(\begin{array}{c}
i+j \\
n+1
\end{array}\right)\right. \\
& \left.+(n-j-1) d\left(\begin{array}{c}
i+j+1 \\
n+1
\end{array}\right)\right] \\
& +\sum_{j=-1}^{n-1} A_{n, j}(a, d)\left[(j+2) d\left(\begin{array}{c}
i+j \\
n+1
\end{array}\right)\right] \\
= & \sum_{j=-1}^{n-1} A_{n, j}(a, d)(a+(n-j-1) d)\left(\begin{array}{c}
i+j+1 \\
n+1
\end{array}\right)
\end{aligned}
$$




$$
\begin{aligned}
& +\sum_{j=-1}^{n-1} A_{n, j}(a, d)(-a+(j+2) d)\left(\begin{array}{c}
i+j \\
n+1
\end{array}\right) \\
& =\sum_{j=-1}^{n}\left[A_{n, j-1}(a, d)(a+(n-j) d)+A_{n, j}(a, d)\right. \\
& \quad \times(-a+(j+2) d)]\left(\begin{array}{c}
i+j \\
n+1
\end{array}\right) \\
& =\sum_{j=-1}^{n} A_{n+1, j}(a, d)\left(\begin{array}{c}
i+j \\
n+1
\end{array}\right) .
\end{aligned}
$$

By (20) With Lemma 7, we can prove the following Lemma which is a generalization of Proposition 5.

Lemma 8. Given an arithmetic progression as in (17),

$$
\begin{aligned}
\sum_{i=1}^{m}(a & +(i-1) d)^{n} \\
& =\sum_{j=-1}^{n-1} A_{n, j}(a, d)\left(\begin{array}{c}
m+j+1 \\
n+1
\end{array}\right) .
\end{aligned}
$$

Proof. We will prove Lemma 8 by induction on $m$.

(i) When $m=1$, by Lemma 7,

$$
\begin{aligned}
(a+(1-1) d)^{n}= & \sum_{j=-1}^{n-1} A_{n, j}(a, d)\left(\begin{array}{c}
1+j \\
n
\end{array}\right) \\
= & \sum_{j=-1}^{n-1} A_{n, j}(a, d)\left(\begin{array}{c}
1+j \\
n
\end{array}\right) \\
& +\sum_{j=-1}^{n-1} A_{n, j}(a, d)\left(\begin{array}{c}
1+j \\
n+1
\end{array}\right),
\end{aligned}
$$

since $\left(\begin{array}{c}1+j \\ n+1\end{array}\right)=0$, for $j=-1,0,1, \ldots, n-1$. Therefore,

$$
[a+(1-1) d]^{n}=\sum_{j=-1}^{n-1} A_{n, j}(a, d)\left(\begin{array}{c}
1+j+1 \\
n+1
\end{array}\right)
$$

which finishes the base case $m=1$.

(ii) Now suppose

$$
\sum_{i=1}^{m}(a+(i-1) d)^{n}=\sum_{j=-1}^{n-1} A_{n, j}(a, d)\left(\begin{array}{c}
m+j+1 \\
n+1
\end{array}\right) .
$$

(iii) Then from Table 3, Definition 6, and Lemma 7,

$$
\begin{aligned}
& \sum_{i=1}^{m+1}(a+(i-1) d)^{n} \\
& \quad=\sum_{j=-1}^{n-1} A_{n, j}(a, d)\left(\begin{array}{c}
m+j+1 \\
n+1
\end{array}\right)+(a+m d)^{n}
\end{aligned}
$$

$$
\begin{aligned}
& =\sum_{j=-1}^{n-1} A_{n, j}(a, d)\left[\left(\begin{array}{c}
m+j+1 \\
n+1
\end{array}\right)+\left(\begin{array}{c}
m+j+1 \\
n
\end{array}\right)\right] \\
& =\sum_{j=-1}^{n-1} A_{n, j}(a, d)\left(\begin{array}{c}
m+1+j+1 \\
n+1
\end{array}\right) .
\end{aligned}
$$

The general Eulerian numbers $A_{n, k}(a, d)$ can be calculated directly from the following formula, which is a generalization of (10).

Lemma 9. For a given arithmetic progression $\{a, a+d, a+$ $2 d, a+3 d, \ldots\}$, the general Eulerian numbers satisfy

$$
A_{n, k}(a, d)=\sum_{i=0}^{k+1}(-1)^{i}[(k+2-i) d-a]^{n}\left(\begin{array}{c}
n+1 \\
i
\end{array}\right) .
$$

Proof. The following proof is given by inductions on both $n$ and $k$.

$$
\begin{aligned}
& \text { For } n=0, k=-1, A_{0,-1}(a, d)=(-1)^{0}(d-a)^{0}\left(\begin{array}{l}
1 \\
0
\end{array}\right)=1 . \\
& \text { For } n=1, k=-1, A_{1,-1}(a, d)=(-1)^{0}(d-a)^{1}\left(\begin{array}{l}
2 \\
0
\end{array}\right)=d-a . \\
& \text { For } n=1, k=0, A_{1,0}(a, d)=(-1)^{0}(2 d-a)^{1}\left(\begin{array}{l}
2 \\
0
\end{array}\right)+ \\
& (-1)^{1}(d-a)^{1}\left(\begin{array}{l}
2 \\
1
\end{array}\right)=a .
\end{aligned}
$$

Now suppose $A_{n-1, k}(a, d)=\sum_{i=0}^{k+1}(-1)^{i}[(k+2-i) d-$ $a]^{n-1}\left(\begin{array}{c}n \\ i\end{array}\right)$. Then from the recursive formula (20),

$$
\begin{aligned}
A_{n, k}(a, d) & \\
= & {[-a+(k+2) d] A_{n-1, k}(a, d) } \\
& +[a+(n-k-1) d] A_{n-1, k-1}(a, d) \\
= & {[-a+(k+2) d] } \\
& \times \sum_{i=0}^{k+1}(-1)^{i}[(k+2-i) d-a]^{n-1}\left(\begin{array}{c}
n \\
i
\end{array}\right) \\
& +[a+(n-k-1) d] \sum_{i=1}^{k+1}(-1)^{i-1} \\
& \times[(k+2-i) d-a]^{n-1}\left(\begin{array}{c}
n \\
i-1
\end{array}\right) \text { by induction } \\
= & {[(k+2) d-a]^{n}\left(\begin{array}{c}
n \\
0
\end{array}\right) } \\
& +\sum_{i=1}^{k+1}(-1)^{i}[(k+2-i) d-a]^{n-1} \\
& \times\left[(k+2) d\left(\begin{array}{c}
n \\
i
\end{array}\right)-(n-k-1) d\left(\begin{array}{c}
n \\
i-1
\end{array}\right)\right. \\
& \left.\quad-a\left(\begin{array}{c}
n \\
i
\end{array}\right)-a\left(\begin{array}{c}
n \\
i-1
\end{array}\right)\right] \\
& {[(k)}
\end{aligned}
$$


TABle 3: First values of general Eulerian numbers $A_{n, k}(a, d)$.

\begin{tabular}{|c|c|c|c|c|c|}
\hline & \multicolumn{5}{|c|}{$k=$} \\
\hline & -1 & 0 & 1 & 2 & 3 \\
\hline$n=0$ & 1 & & & & \\
\hline 1 & $d-a$ & $a$ & & & \\
\hline 2 & $(d-a)^{2}$ & $-2 a^{2}+2 a d+d^{2}$ & $a^{2}$ & & \\
\hline 3 & $(d-a)^{3}$ & $3 a^{3}-6 a^{2} d+4 d^{3}$ & $-3 a^{3}+3 a^{2} d+3 a d^{2}+d^{3}$ & $a^{3}$ & \\
\hline 4 & $(d-a)^{4}$ & $-4 a^{4}+12 a^{3} d-6 a^{2} d^{2}-12 a d^{3}+11 d^{4}$ & $6 a^{4}-12 a^{3} d-6 a^{2} d^{2}+12 a d^{3}+11 d^{4}$ & $-4 a^{4}+4 a^{3} d+6 a^{2} d^{2}+4 a d^{3}+d^{4}$ & $a^{4}$ \\
\hline
\end{tabular}

Note that

$$
\begin{aligned}
(k+2) d\left(\begin{array}{c}
n \\
i
\end{array}\right)-(n-k-1) d\left(\begin{array}{c}
n \\
i-1
\end{array}\right)-a\left(\begin{array}{c}
n \\
i
\end{array}\right)-a\left(\begin{array}{c}
n \\
i-1
\end{array}\right) \\
=(k+2) d\left(\begin{array}{c}
n \\
i
\end{array}\right)-(n-k-1) d\left(\begin{array}{c}
n \\
i-1
\end{array}\right)-a\left(\begin{array}{c}
n+1 \\
i
\end{array}\right) \\
=(k+2) d\left(\begin{array}{c}
n \\
i
\end{array}\right)+(k+2) d\left(\begin{array}{c}
n \\
i-1
\end{array}\right) \\
-(n+1) d\left(\begin{array}{c}
n \\
i-1
\end{array}\right)-a\left(\begin{array}{c}
n+1 \\
i
\end{array}\right) \\
=(k+2) d\left(\begin{array}{c}
n+1 \\
i
\end{array}\right)-i d\left(\begin{array}{c}
n+1 \\
i
\end{array}\right)-a\left(\begin{array}{c}
n+1 \\
i
\end{array}\right) \\
=[(k+2-i) d-a]\left(\begin{array}{c}
n+1 \\
i
\end{array}\right) .
\end{aligned}
$$

Combine the results above, we have

$$
A_{n, k}(a, d)=\sum_{i=0}^{k+1}(-1)^{i}[(k+2-i) d-a]^{n}\left(\begin{array}{c}
n+1 \\
i
\end{array}\right) .
$$

\section{General Eulerian Polynomials}

Definition 10. We define the general Eulerian polynomials associated to an arithmetic progression as in (17) as

$$
T_{n}(t, a, d)=\sum_{k=-1}^{n-1} A_{n, k}(a, d) t^{k+1} .
$$

Definition 10 is a generalization of the traditional Eulerian polynomials as in (6). The following lemma gives the relation between the general Eulerian polynomials and the traditional Eulerian polynomials.

Lemma 11. Let $T_{n}(t, a, d)$ be the general Eulerian polynomials as in Definition 10, $T_{0}(t, a, d)=1$. Then

$$
\begin{aligned}
T_{n}(t, a, d) & =\sum_{k=-1}^{n-1} A_{n, k}(a, d) t^{k+1} \\
& =\sum_{j=0}^{n}\left(\begin{array}{c}
n \\
j
\end{array}\right) d^{j} A_{j}(t)(a t-a)^{n-j},
\end{aligned}
$$

where $A_{j}(t), j=0,1,2, \ldots, n$ are traditional Eulerian polynomials as defined in (4) and (6).

Proof. (i) When $n=0, T_{0}(t, a, d)=\left(\begin{array}{l}0 \\ 0\end{array}\right) d^{0} A_{0}(t)(a t-a)^{0}=1$. (ii) Now suppose

$$
\begin{aligned}
T_{n}(t, a, d)= & \sum_{j=0}^{n}\left(\begin{array}{c}
n \\
j
\end{array}\right) d^{j} A_{j}(t)(a t-a)^{n-j} . \text { Then, } \\
T_{n+1}(t, a, d)= & \sum_{k=-1}^{n} A_{n+1, k}(a, d) t^{k+1} \text { by definition } \\
= & \sum_{k=-1}^{n}(a+(n-k) d) A_{n, k-1}(a, d) t^{k+1} \\
& +\sum_{k=-1}^{n}(-a+(k+2) d) A_{n, k}(a, d) t^{k+1} \\
= & \sum_{k=-1}^{n-1}(a+(n-k-1) d) A_{n, k}(a, d) t^{k+2} \\
& +\sum_{k=-1}^{n-1}(-a+(k+2) d) A_{n, k}(a, d) t^{k+1} \\
= & \sum_{k=-1}^{n-1}(a t-a) A_{n, k}(a, d) t^{k+1} \\
& +\sum_{k=-1}^{n-1} d(n t-t+2) A_{n, k}(a, d) t^{k+1} \\
+ & (a t-a) T_{n}(t, a, d) \\
+ & \sum_{k=-1}^{n-1} d(1-t) k A_{n, k}(a, d) t^{k+1} \\
& \\
& \\
&
\end{aligned}
$$


Note that by definition, $T_{n}(t, a, d)=\sum_{k=-1}^{n-1} A_{n, k}(a, d) t^{k+1}$. So $T_{n}^{\prime}(t, a, d)=\sum_{k=-1}^{n-1}(k+1) A_{n, k}(a, d) t^{k}$, which implies

$$
t T_{n}^{\prime}(t, a, d)=T_{n}(t, a, d)+\sum_{k=-1}^{n-1} k A_{n, k}(a, d) t^{k+1} .
$$

On the other hand, from [3, (3.4), page 263], we have

$$
A_{n}(t)=[1+(n-1) t] A_{n-1}(t)+t(1-t) A_{n-1}^{\prime}(t) .
$$

With results as in (39) and (40), we have

$$
\begin{aligned}
& (d n t-d t+2 d) T_{n}(t, a, d)+\sum_{k=-1}^{n-1} d(1-t) k A_{n, k}(a, d) t^{k+1} \\
& =(d n t+d) T_{n}(t, a, d)+d(1-t) t T_{n}^{\prime}(t, a, d) \\
& =(d n t+d) T_{n}(t, a, d)+d(1-t) t \sum_{j=0}^{n} d^{j}\left(\begin{array}{l}
n \\
j
\end{array}\right) \\
& \times\left[A_{j}^{\prime}(t)(a t-a)^{n-j}\right. \\
& \left.+a(n-j) A_{j}(t)(a t-a)^{n-j-1}\right] \text { by induction } \\
& =(d n t+d) T_{n}(t, a, d)-d t(n-j) T_{n}(t, a, d) \\
& +d(1-t) t \sum_{j=0}^{n}\left(\begin{array}{c}
n \\
j
\end{array}\right) d^{j} A_{j}^{\prime n-j} \\
& =\sum_{j=0}^{n}\left(\begin{array}{c}
n \\
j
\end{array}\right) d^{j+1}(a t-a)^{n-j} \\
& \times\left[(1+j t) A_{j}(t)+t(1-t) A_{j}^{\prime}(t)\right] \\
& =\sum_{j=0}^{n}\left(\begin{array}{c}
n \\
j
\end{array}\right) d^{j+1}(a t-a)^{n-j} A_{j+1}(t) .
\end{aligned}
$$

Therefore, expression (37) becomes

$$
\begin{aligned}
T_{n+1}(t, a, d)= & \sum_{j=0}^{n}\left(\begin{array}{c}
n \\
j
\end{array}\right) d^{j+1}(a t-a)^{n-j} A_{j+1}(t) \\
& +\sum_{j=0}^{n}\left(\begin{array}{c}
n \\
j
\end{array}\right) d^{j}(a t-a)^{n-j+1} A_{j}(t) \\
= & \sum_{j=1}^{n+1}\left(\begin{array}{c}
n \\
j-1
\end{array}\right) d^{j}(a t-a)^{n+1-j} A_{j}(t) \\
& +\sum_{j=0}^{n}\left(\begin{array}{c}
n \\
j
\end{array}\right) d^{j}(a t-a)^{n-j+1} A_{j}(t) \\
= & \sum_{j=0}^{n+1}\left(\begin{array}{c}
n+1 \\
j
\end{array}\right) d^{j} A_{j}(t)(a t-a)^{n+1-j} .
\end{aligned}
$$

The following result is a generalization of (7).

Lemma 12. Let $T_{n}(t, a, d)$ be as defined in Lemma 11. Then

$$
\begin{aligned}
T_{n}(t, u, a, d) & =\sum_{n \geq 0} T_{n}(t, a, d) \frac{u^{n}}{n !} \\
& =\frac{(t-1) \exp (a u(t-1))}{t-\exp (d u(t-1))} .
\end{aligned}
$$

Proof. From Lemma 11

$$
\begin{aligned}
T_{n}(t, u, a, d) & =\sum_{n \geq 0} T_{n}(t, a, d) \frac{u^{n}}{n !} \\
& =\sum_{n \geq 0}\left[\sum_{j=0}^{n}\left(\begin{array}{c}
n \\
j
\end{array}\right) d^{j} A_{j}(t)(a t-a)^{n-j}\right] \frac{u^{n}}{n !} \\
& =\sum_{n \geq 0}\left[\sum_{\substack{k \leq l=n \\
0 \leq k \leq n}} \frac{n !}{k ! l !} d^{k} A_{k}(t)(a t-a)^{l} \frac{u^{k} \cdot u^{l}}{n !}\right] \\
& =\left(\sum_{k \geq 0} A_{k}(t) \frac{(d u)^{k}}{k !}\right)\left(\sum_{l \geq 0} \frac{(u a(t-1))^{l}}{l !}\right) \\
& =\frac{(t-1) \exp (a u(t-1))}{t-\exp (d u(t-1))} .
\end{aligned}
$$

Using the results from Lemma 12, we can derive the following lemma, which is a general version of (5).

Proposition 13. Given an arithmetic progression as in (17), let $T_{n}(t, a-d,-d)$ be the general Eulerian Polynomials associated to the arithmetic progression $\{a-d, a-2 d, a-3 d, \ldots\}$. Then

$$
-\frac{T_{n}(t, a-d,-d)}{(t-1)^{n+1}}=\sum_{j=0}^{\infty} t^{j}(a+j d)^{n} \quad(n \geq 0) .
$$

Proof.

$$
\begin{aligned}
\frac{e^{a u}}{1-t e^{d u}} & =e^{a u} \sum_{j=0}^{\infty}\left(t e^{d u}\right)^{j}=\sum_{j=0}^{\infty} t^{j} e^{u(a+j d)} \\
& =\sum_{j=0}^{\infty} t^{j} \sum_{n=0}^{\infty} \frac{u^{n}(a+j d)^{n}}{n !} \\
& =\sum_{n=0}^{\infty} \frac{u^{n}}{n !}\left(\sum_{j=0}^{\infty} t^{j}(a+j d)^{n}\right) .
\end{aligned}
$$

On the other hand, by Lemma 12,

$$
\begin{aligned}
\sum_{n=0}^{\infty}- & \frac{T_{n}(t, a-d,-d)}{(t-1)^{n+1}} \frac{u^{n}}{n !} \\
& =\frac{1}{t-1} \sum_{n=0}^{\infty}-\frac{T_{n}(t, a-d,-d)}{(t-1)^{n}} \frac{u^{n}}{n !}
\end{aligned}
$$




$$
\begin{aligned}
& =-\frac{1}{t-1} \sum_{n=0}^{\infty} \frac{T_{n}(t, a-d,-d)}{n !}\left(\frac{u}{t-1}\right)^{n} \\
& =-\frac{1}{t-1} \cdot \frac{(t-1) e^{(a-d) u}}{t-e^{-d u}} \\
& =-\frac{e^{(a-d) u}}{t-e^{-d u}}=\frac{e^{a u}}{1-t e^{d u}} .
\end{aligned}
$$

By comparing the coefficients of $u^{n} / n$ !, we have

$$
-\frac{T_{n}(t, a-d,-d)}{(t-1)^{n+1}}=\sum_{j=0}^{\infty} t^{j}(a+j d)^{n} \quad(n \geq 0) .
$$

For the finite summation $\sum_{i=1}^{m} t^{i}[a+(i-1) d]^{n}$, we have the following property which is a generalization of (2) and (3).

Lemma 14. Let $T_{n}$ be the general Eulerian polynomials as defined in Definition 10:

$$
\begin{aligned}
\sum_{i=1}^{m} t^{i} & {[a+(i-1) d]^{n} } \\
= & \sum_{l=0}^{n}\left(\begin{array}{c}
n \\
l
\end{array}\right) \frac{t^{m+1}(d m-d)^{l}}{(t-1)^{n-l+1}} T_{n-l}(t, a,-d) \\
& -\frac{t^{2}}{(t-1)^{n+1}} T_{n}(t, a,-d) \\
\sum_{i=1}^{m} t^{i}[a+(i-1) d]^{n} & \\
= & \frac{t^{m+1}}{(t-1)^{n+1}} T_{n}(t, a+d(m-1),-d) \\
& -\frac{t^{2}}{(t-1)^{n+1}} T_{n}(t, a,-d) .
\end{aligned}
$$

Proof. We will use (2) and (3) to prove expressions (49) and (50), respectively.

$$
\begin{aligned}
\sum_{i=1}^{m} t^{i} & {[a+(i-1) d]^{n} } \\
= & \sum_{i=1}^{m} t^{i}\left[\sum_{j=0}^{n}\left(\begin{array}{c}
n \\
j
\end{array}\right) a^{n-j}(i-1)^{j} d^{j}\right] \\
= & \sum_{j=0}^{n}\left(\begin{array}{c}
n \\
j
\end{array}\right) d^{j} a^{n-j}\left[\sum_{i=1}^{m} t^{i}(i-1)^{j}\right] \\
= & \sum_{j=0}^{n}\left(\begin{array}{c}
n \\
j
\end{array}\right) d^{j} a^{n-j} t\left[\sum_{i=1}^{m-1} i^{j} t^{i}\right] .
\end{aligned}
$$

For (49), if we use (2) to evaluate $\sum_{i=1}^{m-1} i^{j} t^{i}$, we have

$$
\begin{aligned}
& \sum_{i=1}^{m} t^{i}[a+(i-1) d]^{n} \\
& =\sum_{j=0}^{n}\left(\begin{array}{c}
n \\
j
\end{array}\right) d^{j} a^{n-j} t \\
& \times\left[\sum_{l=1}^{j}(-1)^{j+l}\left(\begin{array}{l}
j \\
l
\end{array}\right) \frac{t^{m}(m-1)^{l}}{(t-1)^{j-l+1}} A_{j-l}(t)\right. \\
& \left.+(-1)^{j} \frac{t^{m}-t}{(t-1)^{j+1}} A_{j}(t)\right] \\
& =\sum_{j=0}^{n}\left(\begin{array}{c}
n \\
j
\end{array}\right) d^{j} a^{n-j} t \\
& \times\left[\sum_{l=0}^{j}(-1)^{j+l}\left(\begin{array}{l}
j \\
l
\end{array}\right) \frac{t^{m}(m-1)^{l}}{(t-1)^{j-l+1}} A_{j-l}(t)\right] \\
& +\sum_{j=0}^{n}\left(\begin{array}{c}
n \\
j
\end{array}\right) d^{j} a^{n-j} t(-1)^{j} \frac{-t}{(t-1)^{j+1}} A_{j}(t) \\
& =I+I I \text {; } \\
& I I=\sum_{j=0}^{n}\left(\begin{array}{c}
n \\
j
\end{array}\right) d^{j} a^{n-j} t(-1)^{j} \frac{-t}{(t-1)^{j+1}} A_{j}(t) \\
& =\frac{-t^{2}}{(t-1)^{n+1}} \sum_{j=0}^{n}\left(\begin{array}{c}
n \\
j
\end{array}\right)(-d)^{j}(a(t-1))^{n-j} A_{j}(t) \\
& =-\frac{t^{2}}{(t-1)^{n+1}} T_{n}(t, a,-d) .
\end{aligned}
$$

This gives the second term in (49).

$$
\begin{aligned}
I= & \sum_{j=0}^{n}\left(\begin{array}{c}
n \\
j
\end{array}\right) d^{j} a^{n-j} t \\
& \times \sum_{l=0}^{j}(-1)^{j+l}\left(\begin{array}{l}
j \\
l
\end{array}\right) \frac{t^{m}(m-1)^{l}}{(t-1)^{j-l+1}} A_{j-l}(t) \\
= & \sum_{l=0}^{n} \sum_{j=l}^{n}\left(\begin{array}{c}
n \\
j
\end{array}\right) d^{j} a^{n-j} t(-1)^{j+l}\left(\begin{array}{l}
j \\
l
\end{array}\right) \\
& \times \frac{t^{m}(m-1)^{l}}{(t-1)^{j-l+1}} A_{j-l}(t) \\
= & \sum_{l=0}^{n} \sum_{j=l}^{n} \frac{n !}{j !(n-j) !} d^{j} a^{n-j} t(-1)^{j+l}
\end{aligned}
$$




$$
\begin{aligned}
& \times \frac{j !}{l !(j-l) !} \frac{t^{m}(m-1)^{l}}{(t-1)^{j-l+1}} A_{j-l}(t) \\
= & \sum_{l=0}^{n} \sum_{k=0}^{n-l} \frac{n !}{(n-k-l) !} d^{k+l} a^{n-k-l} t(-1)^{k} \\
& \times \frac{1}{l ! k !} \frac{t^{m}(m-1)^{l}}{(t-1)^{k+1}} A_{k}(t) \quad k=j-l \\
= & \sum_{l=0}^{n} \frac{(m-1)^{l} n ! t^{m+1} d^{l}}{l !(n-l) !} \\
& \times \sum_{k=0}^{n-l} \frac{(-d)^{k} a^{n-k-l}(n-l) !}{k !(n-k-l) !(t-1)^{k+1}} A_{k}(t) \\
= & \sum_{l=0}^{n}\left(\begin{array}{c}
n \\
l
\end{array}\right) \frac{(m-1)^{l} t^{m+1} d^{l}}{(t-1)^{n-l+1}} \\
= & \sum_{l=0}^{n}\left(\begin{array}{l}
n \\
l
\end{array}\right) \frac{\sum^{m+1}(d m-d)^{l}}{(t-1)^{n-l+1}} T_{n-l}(t, a,-d), \\
& \left(\begin{array}{c}
n-l \\
k
\end{array}\right)(-d)^{k}(a t-a)^{n-k-l} A_{k}(t)
\end{aligned}
$$

which gives the first term of (49). So we have proved (49) by using expression (2).

For (50), if we use (3) to evaluate $\sum_{i=1}^{m-1} i^{j} t^{i}$, we have

$$
\begin{aligned}
& \sum_{i=1}^{m} t^{i}[a+(i-1) d]^{n} \\
& =\sum_{j=0}^{n}\left(\begin{array}{c}
n \\
j
\end{array}\right) d^{j} a^{n-j} t \\
& \times\left[-t^{m} \sum_{k=0}^{j}\left(\begin{array}{l}
j \\
k
\end{array}\right) \frac{(m-1)^{j-k}}{(1-t)^{k+1}} A_{k}(t)\right. \\
& \left.+\frac{t}{(1-t)^{j+1}} A_{j}(t)\right] \\
& =-t^{m+1} \sum_{j=0}^{n}\left(\begin{array}{l}
n \\
j
\end{array}\right) d^{j} a^{n-j} \sum_{k=0}^{j}\left(\begin{array}{l}
j \\
k
\end{array}\right) \frac{(m-1)^{j-k}}{(1-t)^{k+1}} A_{k}(t) \\
& +\sum_{j=0}^{n}\left(\begin{array}{c}
n \\
j
\end{array}\right) d^{j} a^{n-j}(1-t)^{-j} \frac{t^{2}}{1-t} A_{j}(t) \\
& =I I I+I V \text {; } \\
& I V=\sum_{j=0}^{n}\left(\begin{array}{c}
n \\
j
\end{array}\right) d^{j} a^{n-j}(1-t)^{-j} \frac{t^{2}}{1-t} A_{j}(t)
\end{aligned}
$$

$$
\begin{aligned}
& =\sum_{j=0}^{n}\left(\begin{array}{c}
n \\
j
\end{array}\right)(-d)^{j} a^{n-j}(t-1)^{n-j} \frac{t^{2}}{(1-t)(t-1)^{n}} A_{j}(t) \\
& =-\frac{t^{2}}{(t-1)^{n+1}} T_{n}(t, a,-d),
\end{aligned}
$$

by Lemma 11. Thus, we have obtained the second part of (50).

$$
\begin{aligned}
& I I I=-t^{m+1} \sum_{j=0}^{n}\left(\begin{array}{l}
n \\
j
\end{array}\right) d^{j} a^{n-j} \\
& \times \sum_{k=0}^{j}\left(\begin{array}{l}
j \\
k
\end{array}\right) \frac{(m-1)^{j-k}}{(1-t)^{k+1}} A_{k}(t) \\
& =-t^{m+1} \sum_{j=0}^{n} \sum_{k=0}^{j} \frac{n !}{j !(n-j) !} \cdot \frac{j !}{k !(j-k) !} \\
& \times d^{j} a^{n-j} \frac{(m-1)^{j-k}}{(1-t)^{k+1}} A_{k}(t) \\
& =-t^{m+1} \sum_{k=0}^{n} \frac{n !}{k !(1-t)^{k+1}} A_{k}(t) \\
& \times \sum_{j=k}^{n} \frac{1}{(n-j) !(j-k) !}(m-1)^{j-k} d^{j} a^{n-j} \\
& =-t^{m+1} \sum_{k=0}^{n} \frac{n !}{k !(1-t)^{k+1}} A_{k}(t) \\
& \times \sum_{l=0}^{n-k} \frac{1}{l !(n-k-l) !}(m-1)^{l} d^{l+k} a^{n-l-k} \quad l=j-k \\
& =-t^{m+1} \sum_{k=0}^{n}\left(\begin{array}{l}
n \\
k
\end{array}\right) \frac{d^{k}}{(1-t)^{k+1}} A_{k}(t)(a+d(m-1))^{n-k} \\
& =\frac{t^{m+1}}{(t-1)^{n+1}} \sum_{k=0}^{n}\left(\begin{array}{l}
n \\
k
\end{array}\right)(-d)^{k}(t-1)^{n-k} \\
& \times A_{k}(t)(a+d(m-1))^{n-k} \\
& =\frac{t^{m+1}}{(t-1)^{n+1}} T_{n}(t, a+d(m-1),-d),
\end{aligned}
$$

which gives the first term of (50).

\section{Acknowledgments}

The authors want to express their deep appreciation to Professor Dominique Foata and the referee for their kind help. 


\section{References}

[1] J. Bernoulli, Ars conjectandi, opus posthumum. Accedit tractatus de seriebus infinitis, et epistola gallicè scripta de ludo pilae reticularis, Impensis Thurnisiorum, fratrum, Basel, Switzerland, 1713.

[2] L. Euler, Institutiones calculi differentialis cum eius usu in analysi finitorum ac Doctrina serierumm, chapter VII, Academiae Imperialis Scientiarum Petropolitanae, Petersburg, Russia, 1755.

[3] D. Foata, "Eulerian polynomials: from Euler's time to the present," in The Legacy of Alladi Ramakrishnan in the Mathematical Sciences, pp. 253-273, Springer, New York, NY, USA, 2010.

[4] L. Euler, "Methodus universalis series summandi ulterius promota," in Commentarii academia scientiarum imperialis Petropolitana 8, pp. 147-158, Academiae scientiarum imperialis Petropolitanae, 1736.

[5] L. Carlitz, " $q$-Bernoulli and Eulerian numbers," Transactions of the American Mathematical Society, vol. 76, pp. 332-350, 1954.

[6] L. Carlitz, "A combinatorial property of $q$-Eulerian numbers," The American Mathematical Monthly, vol. 82, pp. 51-54, 1975.

[7] J. Worpitzky, "Studien uber die Bernoullischen und Eulerischen Zahlen," Journal für die reine und angewandte Mathematik, vol. 94, pp. 203-232, 1883.

[8] H. Tsao, "Sums of powers and Eulerian numbers," The Mathematical Gazette, vol. 95, pp. 347-349, 2011. 


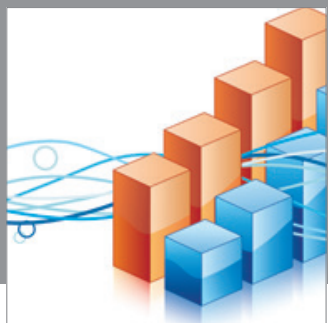

Advances in

Operations Research

mansans

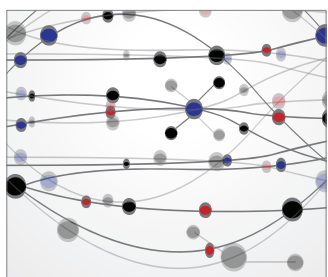

The Scientific World Journal
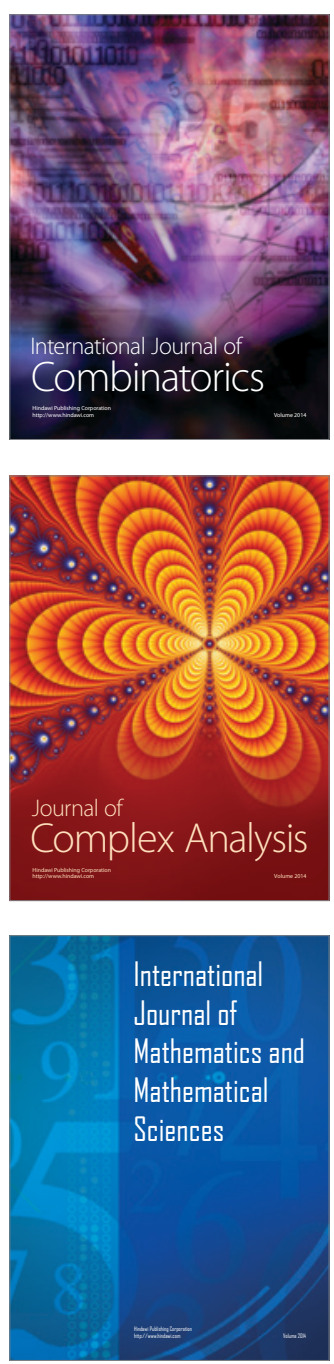
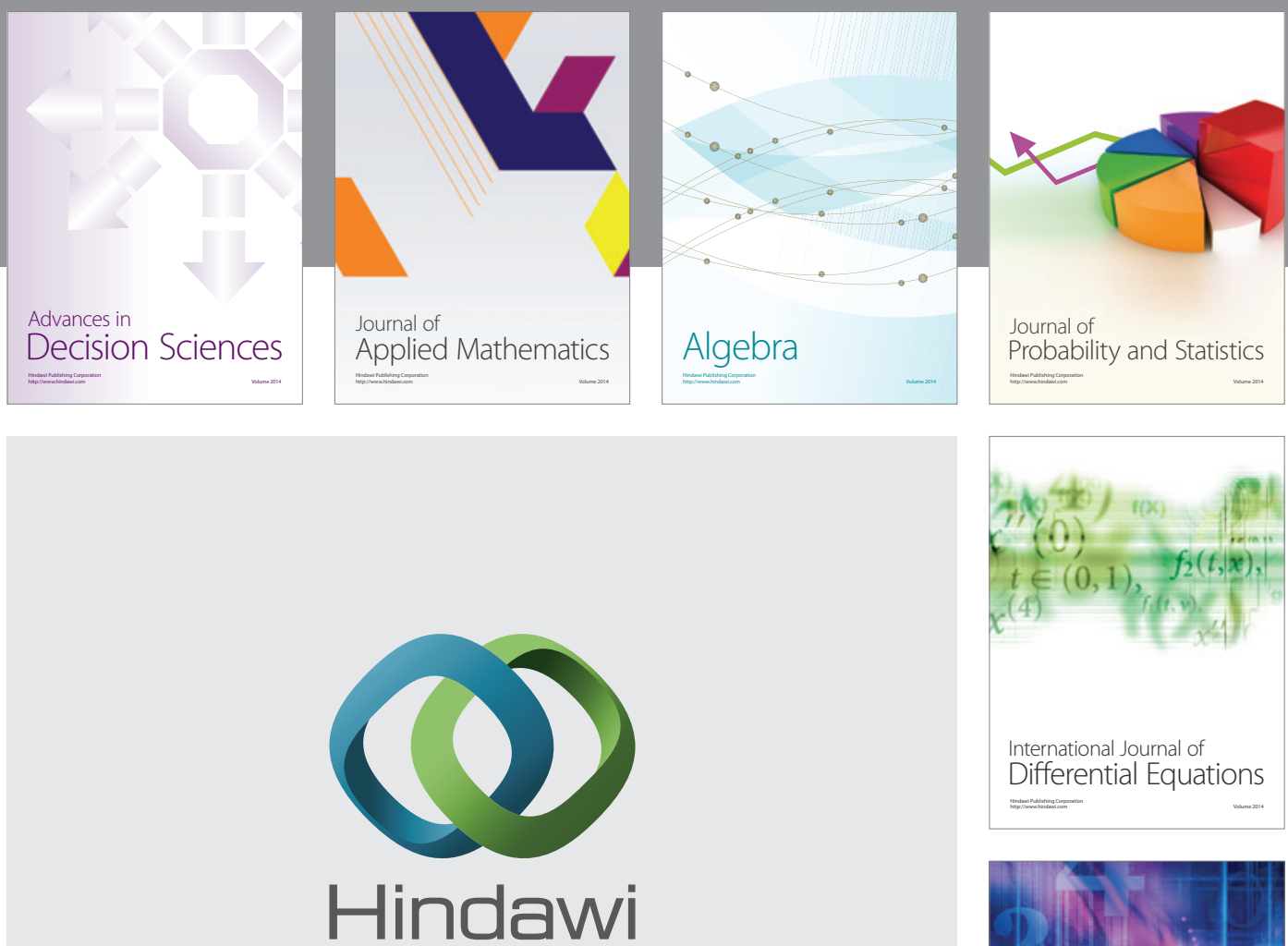

Submit your manuscripts at http://www.hindawi.com
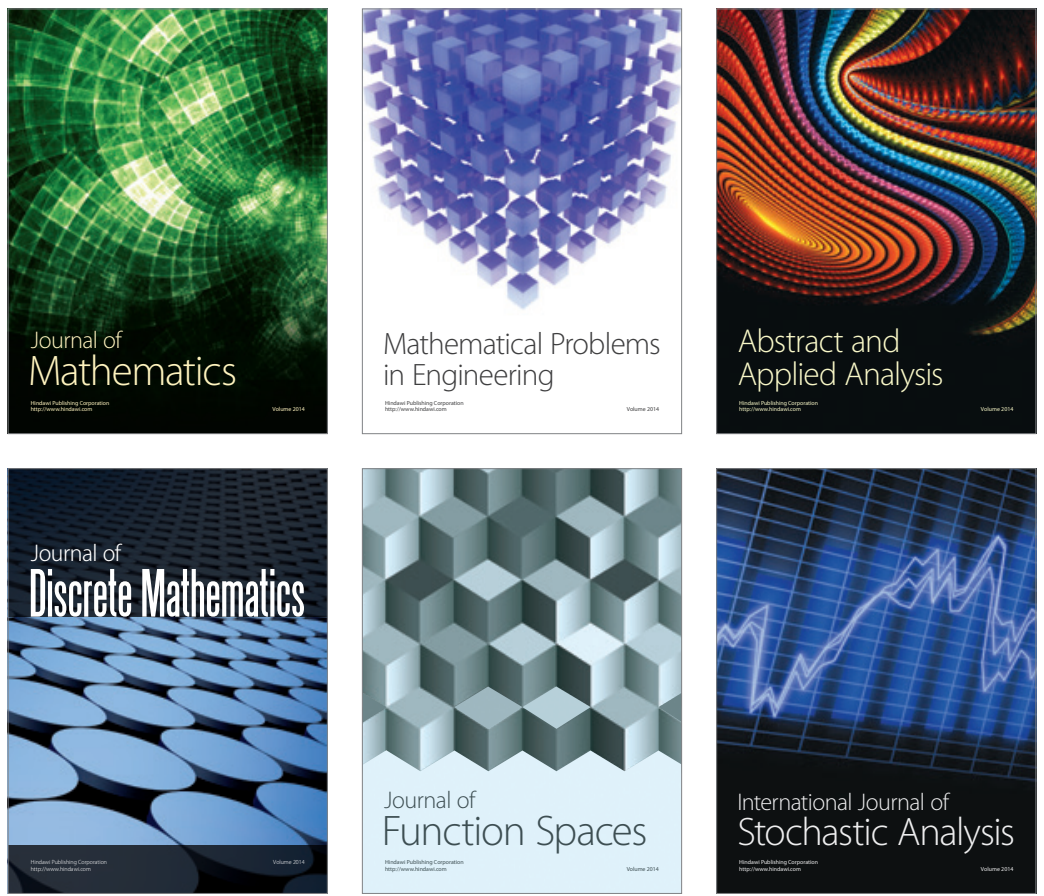

Journal of

Function Spaces

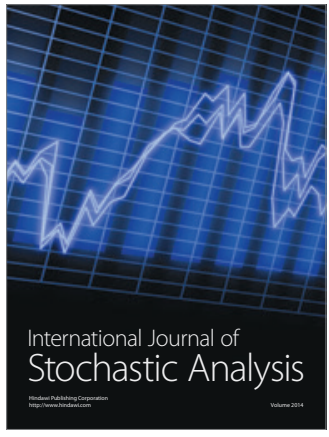

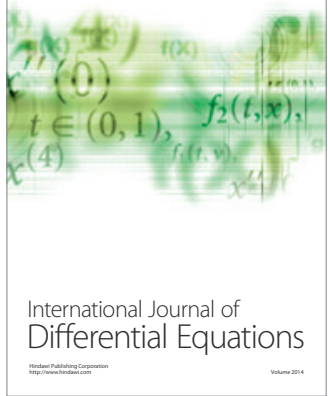
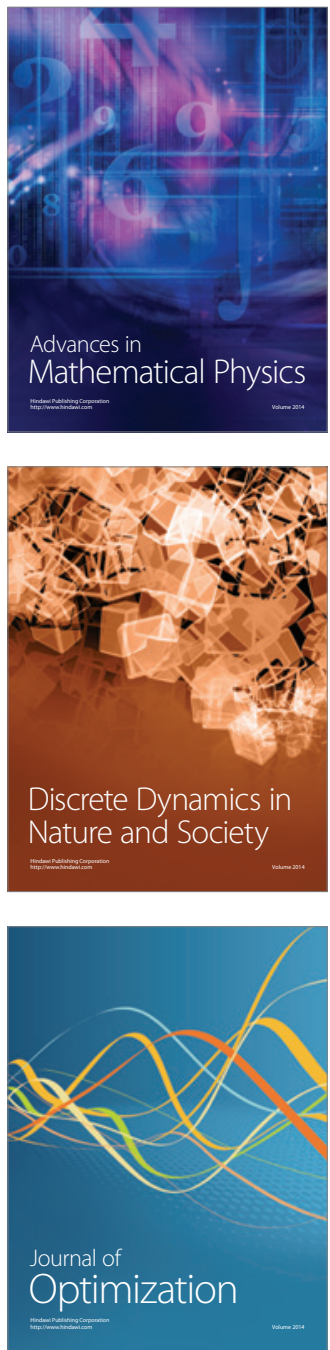